\title{
IMPORTÂNCIA DA VITAMINA C NO CRESCIMENTO E DESENVOLVIMENTO GONADAL EM PEIXES
}

\author{
MARTINS, Tamyres Pereira Araújo ${ }^{1}$ \\ FERREIRA, Thalita Milo Simões ${ }^{1}$ \\ GOMIDES, Pedro Fellipe Vieira ${ }^{3}$ \\ NAVARRO, Fernanda Keley Silva Pereira ${ }^{4}$ \\ MURATA, Luci Sayori ${ }^{5}$ \\ NAVARRO, Rodrigo Diana ${ }^{2}$
}

RESUMO: O crescimento saudável dos peixes bem como, a melhora nos índices reprodutivos, é alcançado com o balanceamento de dietas em função da exigência nutricional em cada fase de desenvolvimento. A vitamina $\mathrm{C}$ atua em diversos processos fisiológicos no peixe, sendo, portanto, de suma importância o conhecimento dos seus efeitos no organismo do animal. O presente trabalho aborda os efeitos da vitamina $\mathrm{C}$ no crescimento e no desenvolvimento gonadal dos peixes.

Palavras-Chave: Crescimento. Desenvolvimento gonadal. Peixe. Reprodução.

\section{IMPORTANCE OF VITAMIN C ON GROWTH AND GONADAL DEVELOPMENT IN FISH}

SUMMARY: The healthy growth of fish as well as the improvement in reproductive rates is achieved by balancing diets according to the nutritional requirements at each stage of development. Vitamin $\mathrm{C}$ acts in several physiological processes in fish, and is therefore of paramount importance to study their effects on the body of the animal. This paper discusses the effects of vitamin $\mathrm{C}$ on growth and gonadal development of fish.

Keyword: Fish. Gonadal development. Growth. Reproduction.

\section{INTRODUÇÃO}

O crescimento e desenvolvimento saudável dos animais dependem do fornecimento de uma dieta que atenda suas exigências nutricionais (NAVARRO et al., 2010). Em criações extensivas os peixes selecionam alimentos que apresentam composição nutricional próxima ás suas exigências, variando em função do seu hábito alimentar. $\mathrm{O}$ alimento natural tem como principal componente os plânctons. Estes apresentam alto valor nutritivo, sendo ricos em

\footnotetext{
${ }^{1}$ Bolsista Proic/CNPq, Grupo de pesquisa em aquicultura AcquaUnB da Universidade de Brasília, Campus Universitário Darcy Ribeiro, Caixa Postal 4.508, CEP 70910-970 Brasília, DF. E-mail: tamyresmartins@zootecnista.com.br

${ }^{2}$ Professor de aquicultura da Universidade de Brasília, Campus Universitário Darcy Ribeiro, Caixa Postal 4.508, CEP 70910-970 Brasília, DF. E-mail: navarrounb@gmail.com

${ }^{3}$ Professor de piscicultura da UPIS, Fazenda Lagoa Bonita, BR-020, DF-335, km 4,8 - Planaltina-DF. E-mail: gorfellipe@hotmail.com

${ }^{4}$ Bióloga, Instituto de Ciência Biológica da Universidade de Brasília. Campus Universitário Darcy Ribeiro, Caixa Postal 4.508, CEP 70910-970 Brasília, DF

${ }^{5}$ Professor da Universidade de Brasília, Campus Universitário Darcy Ribeiro, Caixa Postal 4.508, CEP 70910-970 Brasília, DF.
} 
minerais, vitaminas, energia e em proteína de alta qualidade (FURUYA et al., 1999). Nos sistemas intensivos e superintensivos o alimento natural é restrito e o suprimento das exigências nutricionais provém do alimento fornecido, o qual deve conter ingredientes de alta digestibilidade, perfil nutricional de alta biodisponibilidade e em proporções adequadas para o máximo aproveitamento pelo animal (ALMEIDA, 2003). A produtividade do animal pode ser comprometida pelo mau armazenamento da ração, pela biodisponibilidade dos nutrientes e pela concentração de vitaminas e minerais (CAVALHEIRO, 1998).

$\mathrm{O}$ alimento é constituído basicamente por água e compostos orgânicos e minerais. As substâncias orgânicas diferem entre si por suas propriedades físico-químicas e biológicas, sendo classificadas em carboidratos, proteínas, lipídeos e vitaminas (NUNES, 1998).

A proteína é o principal nutriente exigido pelo organismo animal para o crescimento e manutenção dos tecidos dos órgãos vitais, sendo exigida pelos peixes em quantidades elevadas para atender ás exigências de manutenção e produção (FURUYA, 2010). A deficiência proteica na dieta pode reduzir o crescimento dos peixes e os aminoácidos dos tecidos musculares podem ser degradados para manutenção dos tecidos vitais, em contrapartida, o excesso de proteína aumenta o gasto energético para a excreção de resíduos nitrogenados (ALMEIDA, 2003). O nível ideal de proteína dietética é influenciado pela fase de desenvolvimento e hábito alimentar do animal, pela densidade de estocagem, temperatura da água e pela digestibilidade proteica (PIEDRAS et al., 2004)

Os nutrientes além de fornecerem elementos essenciais ao organismo animal também fornecem energia para manutenção dos processos metabólicos (NUNES, 1998). Dentre os nutrientes exigidos pelos peixes, os lipídeos são os que mais fornecem energia, além de serem constituintes das membranas celulares, precursores de hormônios esteroides (MEURER et al., 2002) e auxiliarem no transporte de vitaminas lipossolúveis para o enterócito (ALMEIDA, 2003).

Peixes onívoros, comparados aos carnívoros, possuem melhor capacidade de conversão de carboidratos em energia. A inclusão dessas macromoléculas á dieta substitui os níveis de lipídeos e proteínas utilizados para produção de energia, destinando os aminoácidos para o crescimento do animal (ALMEIDA, 2003).

Os Minerais estão presentes em pequenas quantidades nos alimentos e são exigidos em pequenas quantidades pelo animal (COLOMBANO et al., 2007). São classificados em macrominerais (cálcio, fósforo, sódio, cloro, potássio, magnésio e enxofre) e microminerais (ferro, iodo, cobre, flúor, manganês, molibdênio, zinco, cobalto, selênio, cromo e silício) em função da quantidade do elemento presente no organismo. Os minerais atuam como constituintes das estruturas esqueléticas, regulam o equilíbrio ácidobásico e a pressão osmótica e, atuam como ativadores de enzimas. Tanto o excesso quanto a deficiência mineral podem provocar alterações 
patológicas específicas para cada mineral (NUNES, 1998). No entanto, a suplementação de minerais à dieta potencializa a ação de algumas vitaminas e maximiza o crescimento dos animais (ALMEIDA, 2003).

O ácido ascórbico (vitamina C) atua em diversos processos metabólicos (FUJIMOTO et $a l .$, 2013), sendo considerado um nutriente essencial e indispensável para a maioria das espécies de peixes (BARAÚNA et al., 2009).

Nos sistemas de cultivo intensivo, pela limitação de alimento natural (NAVARRO et al., 2009), pode ser facilmente observada a deficiência de vitamina C (PEZZATO, 1999), caracterizada, principalmente, pela redução no crescimento dos peixes e por deformidades esqueléticas (MIRANDA et al., 2003; ZHOU et al. 2012), sendo necessária a suplementação da vitamina à dieta para reduzir ou prevenir os sinais de deficiência (BARAÚNA et al., 2009).

Objetivou-se com esta revisão, abordar os efeitos da vitamina C sobre o crescimento e desenvolvimento gonadal dos peixes.

\section{VITAMINAS}

Vitaminas são compostos orgânicos presentes em quantidades diminutas nos alimentos (COMBS JR., 2012), são essenciais para manutenção de diversos processos bioquímicos e fisiológicos (NEU et al., 2010) atuando como cofatores ou substratos em algumas reações metabólicas e não são sintetizadas pelo organismo animal ou o são em quantidades insuficientes, sendo necessário o fornecimento exógeno (MIRANDA et al., 2003). Diferem dos carboidratos, lipídeos e proteínas por não desempenhar função estrutural e por não produzirem energia (NUNES, 1998). São classificadas em lipossolúveis (A, D, E e K) e hidrossolúveis (vitaminas do complexo B e ácido ascórbico) (BORBA et al., 2013).

As vitaminas lipossolúveis, com exceção das vitaminas A e $K$, não atuam como coenzimas e são armazenadas no fígado e tecido adiposo sendo disponibilizadas de acordo com a necessidade do animal (COMBS JR., 2012). Já as do grupo hidrossolúvel (tiamina, riboflavina, piridoxina, ácido pantotênico, niacina, biotina, ácido fólico, cianocobalamina, colina, inositol e ácido ascórbico) atuam como coenzima no metabolismo celular (NRC, 2011); são armazenadas em pequenas quantidades no organismo e rapidamente excretadas sendo necessário o fornecimento constante por via dietética (BORBA et al., 2013).

As exigências em vitaminas podem ser afetadas por fatores intrínsecos ao animal (taxa de crescimento, tamanho corporal e espécie), pela composição da dieta e interação com os outros nutrientes e ainda pelo sistema de produção (ALMEIDA, 2003). 


\section{VITAMINA C}

Todos os compostos que exprimam a atividade biológica do ácido ascórbico são genericamente descrito como vitamina $\mathrm{C}$, sendo a forma mais ativa, biologicamente, o ácido Lascórbico (COMBS JR.,2012).

Durante o processamento e estocagem de rações a forma ativa da vitamina $\mathrm{C}$ pode ser facilmente oxidada para a forma inativa (ácido dicetogulônico). Fatores como a umidade, temperaturas elevadas, incidência direta de luz e a presença de metais pesados, são os principais responsáveis pela inativação do ácido ascórbico (ROTTA, 2003). Assim, recomenda-se o uso de formas protegidas de ácido ascórbico que, por serem mais estáveis, são mais resistentes ao processamento como os derivados fosforados de ácido ascórbico (ascorbil polifosfato) (BORBA et al., 2013).

O ácido ascórbico é o agente redutor mais potente disponível às células, atuando como antioxidante, pelo alto potencial de redução (ROTTA, 2003). Participa como cofator nas reações de hidroxilação para a síntese de carnitina, norepinefrina (SOLIMAN et al., 1986; JIMÉNEZFERNÁNDEZ et al., 2012), colágeno (ZHOU et al. 2013) e além de manter a integridade da membrana celular impedindo sua peroxidação lipídica em ação sinérgica com a vitamina $\mathrm{E}$ (NRC, 2011; GAO, 2014).

\section{VITAMINA C NO CRESCIMENTO}

A maioria dos animais é capaz de sintetizar ácido ascórbico, entretanto, algumas espécies de peixes, camarões, porcos da Índia e o homem não são capazes de sintetizá-lo (NRC, 2011) devido à ausência (NAVARRO, 2010) ou inatividade da enzima L-gulonolactona oxidase que catalisa a última reação da síntese da vitamina C a partir da glucose (SHIAU \& HSU, 1999;).

Em dietas deficientes em vitamina $\mathrm{C}$, têm sido observados diversos sinais clínicos como redução no crescimento, principalmente nas fases iniciais (MIRANDA et al., 2003), deformidades na coluna vertebral, erosão da nadadeira caudal (ZHOU et al. 2012), deformidades nas cartilagens das brânquias e no opérculo, deficiência na cicatrização de feridas, perda de escamas, (ROTTA, 2003; NEU et al., 2010; BORBA et al., 2013), diminuição da eclodibilidade, hemorragias, anemia, exoftalmia e letargia (SOLIMAN et al., 1986; NRC, 2011). Navarro et al., (2009) salienta que o escorbuto é comumente encontrado em sistemas intensivos de produção, caracterizado pelas altas densidades e pela limitação de alimento natural.

O ácido ascórbico tem participação diretamente no crescimento dos peixes por participar na formação de colágeno, principal componente do esqueleto, mantendo a atividade da enzima hidroxilase na reação de hidroxilação de lisina e prolina no protocolágeno (ROTTA, 2003; 
FUJIMOTO et al., 2013), sendo, portanto, necessário para o desenvolvimento normal do animal (NEU et al., 2010).

Chagas e Val (2003) relataram que juvenis de tambaqui (Colossoma macropomum) apresentaram melhor ganho de peso, conversão alimentar e sobrevivência, quando receberam 100mg de ácido L-ascórbico/kg de ração.

Trabalhando com larvas Rhamdia voulezi, Reis et al. (2011) constataram que a suplementação de $600 \mathrm{mg} / \mathrm{Kg}$ de ácido ascórbico á dieta apresentou aumento no desempenho produtivo e melhora na taxa de sobrevivência.

Navarro et al. (2010), trabalhando com suplementação de vitamina C na dieta de póslarvas de Oreochromis niloticus, observaram melhora na conversão alimentar aparente, refletindo em maior ganho em peso, sugerindo a participação do ácido ascórbico na síntese de colágenoe, consequentemente, no crescimento do peixe. Resultado semelhante foi encontrado no estudo de Ibrahem et al. (2010) em alevinos de Tilápias-do Nilo suplementadas com ácido ascórbico. Entretanto, estes resultados divergem do estudo de Barros et al.(2002) que não encontraram efeitos significativos em parâmetros de desempenho produtivo de tilápias-do-Nilo, suplementadas com diferentes níveis de vitamina $\mathrm{C}$ e ferro à dieta. Provavelmente a forma do ácido ascórbico utilizada interferiu nos resultados.. Borba et al. (2013) recomendam o uso de formas protegidas de ácido ascórbico por serem mais estáveis.

\section{VITAMINA C NO DESENVOLVIMENTO GONADAL}

O crescimento, a reprodução, a saúde e o metabolismo dos peixes requerem pequenas quantidades de vitaminas. Portanto, à formulação de dietas para reprodutores, devem ser consideradas as exigências vitamínicas do peixe, uma vez que, a composição de vitaminas poderá refletir no desenvolvimento gonadal, na qualidade e quantidade de ovócitos e espermatozoides (NAVARRO et al., 2006a), bem como no desenvolvimento embrionário e na sobrevivência de larvas (FERNANDEZ-PALÁCIO et al., 1998).

A ligação da vitamina $C$ com a reprodução está na sua participação na vitelogênese e na embriogênese (ROTTA, 2003). Estudos demonstram que o desempenho reprodutivo das fêmeas diminui quando são fornecidas dietas isentas ou com baixa quantidade de ácido ascórbico, provocando uma diminuição de disponibilidade de vitamina $\mathrm{C}$ no ovário, reduzindo o número de ovos e a eclodibilidade e, aumentando tanto o número de larvas com deformidade, quanto a sua mortalidade (SOLIMAN et al., 1986; NAVARRO et al., 2009).

Alguns nutrientes, inclusive o ácido ascórbico, são transferidos dos reprodutores para o embrião. Por esta razão a dieta dos reprodutores não deve atender somente suas exigências nutricionais, mas também o desenvolvimento embrionário após a desova (ROTTA, 2003). 
Os índices gonadossomático (IGS) e hepatossomático (IHS) são parâmetros usados, frequentemente, para avaliar os efeitos da nutrição na reprodução. Conforme descrito por Navarro et al. (2009), o índice gonadossomático expressa o percentual de massa da gônada (testículo ou ovário) em relação ao peso corporal, sendo um importante parâmetro de avaliação de atividade reprodutiva, associado à maturidade e fecundidade dos indivíduos. Desta forma, quanto maior o peso das gônadas, maior é a produção de gametas (NAVARRO et al., 2006b).

$\mathrm{O}$ ácido ascórbico tem a função de regenerar o $\alpha$ - tocoferol (genericamente denominado de vitamina $\mathrm{E}$ ) em sua forma reduzida (NRC, 2011). Atuam de forma sinérgica prevenindo danos às membranas celulares dos ovócitos e dos espermatozoides da ação oxidativa dos radicais livres (GAO, 2014) e, consequentemente, segundo Navarro et al. (2006b), aumentam o número de gametas que reflete no peso das gônadas.

Lee e Dabrowski (2004) observaram que a combinação de $160 \mathrm{mg} / \mathrm{kg}$ de vitamina E e 250 $\mathrm{mg} / \mathrm{kg}$ de vitamina $\mathrm{C}$ resultou em melhor qualidade de sêmen em "Yellow perch" Perca flavescens.

Navarro et al. (2010) observaram que tilápias-do Nilo suplementadas com $50 \mathrm{mg} / \mathrm{kg}$ de vitamina $\mathrm{C}$ na dieta, apresentaram melhores porcentagens de células de Leydig, sugerindo relação funcional do ácido ascórbico com o processo reprodutivo

\section{CONCLUSÃO}

$\mathrm{O}$ uso da vitamina $\mathrm{C}$ na dieta apresenta bons resultados no crescimento e no desenvolvimento gonadal de alguns peixes. No entanto, em sistemas intensivos, torna-se necessário a suplementação dessa vitamina nas dietas de algumas espécies desses vertebrados aquáticos devido a sua incapacidade de sintetizar vitamina C. Assim, essas informações poderão nortear novas pesquisas, melhorar as condições de cultivo em sistema intensivos, valorizar a atividade aumentando a qualidade e quantidade de alevinos destinados à aquicultura.

\section{REFERÊNCIA}

ALMEIDA, G.S.C. Suplementação dietética de vitamina C, desenvolvimento e sanidade do Pacu (Piaractus mesopotamicus). 47 pp. 2003. (Dissertação de mestrado) Escola Superior de Agricultura Luis de Queiroz. Piracicaba.

BARAUNA, L.C.R.I.et al. Utilização de duas diferentes megadoses de vitamina C na ração de alevinos de tilápia do Nilo (Oreochromis niloticus) submetidos à infecção experimental por Edwardsiella tarda. Arq. Inst. Biol., São Paulo, v.76, n.4, p.561-567.2009

BARROS, M.M.et al. níveis de vitamina C e ferro para tilápia do Nilo (oreochromis niloticus). Revista Brasileira de Zootecnia, v.31, n.6, p. 2149-2156, 2002. 
BORBA, M.R.D.; E SÁ, M.V.C.; DE ABREU, J.S. Vitaminas e Minerais. In: Nutriaqua: Nutrição e alimentação de espécies de interesse para a aquicultura brasileira. Florianópolis: Aquabio, 2013. p. 121-167.

CAVALHEIRO, J.M.O.; PEREIRA, J.A. Efeito de níveis de proteína e energia em dietas no crescimento do robalo, Centropomus parallelus (Poey, 1860) em água doce, In: Anais do Congresso Aquicultura Brasil' 98, Anais... v.2. p35-40 1998.

CHAGAS, E.C. \& VAL, A.L. Efeito da vitamina C no ganho de peso e em parâmetros hematológicos de tambaqui. Pesquisa Agropecuária Brasileira, Brasília, v.38, n.3, p. 397-402, 2003.

COLOMBANO, N.C.et al. Suplementação alimentar com vitamina c e desempenho zootécnico de girinos de rã-touro (Rana catesbeiana). Acta Scientiarum. Animal Sciences, v. 29, n. 3, p. 333-338, 2007.

COMBS Jr., G.F. The vitamins: fundamental aspects in nutrition and health. 4ed. New York: Academic Press, 598p. 2012.

FERNANDEZ-PALACIOS, H.et al. Combined effect of dietary a-tocopherol and ny3 HUFA on egg quality of gilthead seabream broodstock Sparus aurata . Aquaculture, v.161, p.475-476, 1998.

FUJIMOTO, R.Y.; SANTOS, R.F.B.; CARNEIRO, D.J. Morphological deformities in the osseous structure in spotted sorubim Pseudoplatystoma coruscans (agassiz \& spix, 1829) with vitamin C deficiency, In: Anais da Academia Brasileira de Ciências (2013) 85(1): 379-384.

FURUYA, V.R.B.et al. Influência de plâncton, dieta artificial e sua combinação, sobre o crescimento e sobrevivência de larvas de curimbatá (Prochilodus lineatus). Acta Scientiarum, v. 21, n.3, p. 699-703, 1999.

FURUYA, W.M. (Ed.). Tabelas brasileiras para a nutrição de tilápias. GFM, 2010.

GAO, J.et al. Interactive effects of vitamin C and E supplementation on growth performance, fatty acid composition and reduction of oxidative stress in juvenile Japanese flounder Paralichthys olivaceus fed dietary oxidized fish oil. Aquaculture, v. 422, p. 84-90, 2014.

IBRAHEM, M.D.et al.. Effect of dietary supplementation of inulin and vitamin $\mathrm{C}$ on the growth, hematology, innate immunity, and resistance of Nile tilapia (Oreochromis niloticus). Fish and Shellfish Immunology, v. 29, p. 241-246, 2010.

JIMÉNEZ-FERNÁNDEZ, E.et al.. Molecular characterization and transcriptional regulation of the sodium-dependent vitamin $\mathrm{C}$ transporter genes (slc23a1 and slc23a2) in a teleost fish, the Senegalese sole (Solea senegalensis). Comparative Biochemistry and Physiology, Part B, v. 161, p. 208-218, 2012.

LEE, K.J; DABROWSKI, K. Long-term effects and interacions of dietary vitamins C and E on growth and reproduction of yellow perch, Perca flavescens. Aquaculture. V 230, p. 377-389, 2004.

MEURER, F.et al. Lipídeos na Alimentação de Alevinos Revertidos de Tilápia do Nilo (Oreochromis niloticus, L.). Revista Brasileira de Zootecnia, v. 31, n. 2, p. 566-573, 2002. 
MIRANDA, E. C.et al. Ganho de peso e taxa de sobrevivência de pós-larvas de pacu (Piaractus mesopotamicus) alimentadas com rações contendo diferentes níveis de vitamina C. Acta

Scientiarum. Animal Sciences, v. 25, n. 1, p. 31-36, 2003.

NATIONAL RESEARCH COUNCIL. (NRC). Nutrient Requirements of Fish and Shrimp., Washington, D.C., USA: National Academy Press, 2011.

NAVARRO, R.D.et al. Níveis de energia digestível na dieta de piauçu no desenvolvimento testicular em estágio pós-larval. Zootec Trop, v.24, p.153-163, $2006 a$.

NAVARRO, R.D.et al. Comparação morfométrica e índices somáticos de machos e fêmeas do lambari prata (Astayanax scabripinnis Jerenyns, 1842) em diferente sistema de cultivo. Zootec Trop, v.24, p.22-33, 2006b.

NAVARRO, R.D.et al._A Importância da vitamina E, C e A na reprodução de peixes (Revisão de literatura). Revista Brasileira de Reprodução Animal, v. 33, p. 20-25, 2009.

NAVARRO, R.D.et al. Morformetria e desenvolvimento gonadal em Tilápia do Nilo (Oreochromis niloticus) alimentadas com suplementação de vitaminas E. Archivos de Zootecnia, v. 59, p. 519-528, 2010.

NEU, D.H. et al. Suplementação de vitamina C na dieta para larvas de Mandi-pintado Pimelodus britskii. Acta Veterinaria Brasilica, v. 4, n. 4, p. 242 - 246, 2010.

NUNES, I. J. Nutrição Animal Básica. Belo Horizonte: FEP-MVZ, 1998, 388 p.

PEZZATO, L.E. 1999, Alimentação de peixes relação custo e benefício. In: REUNIÃO ANUAL SOCIEDADE BRASILEIRA DE ZOOTECNIA, 36, Anais... 26 a 29 de julho de 1999, p. 109 118.

PIEDRAS, S.R.N.; POUEY, J.L.O.F.; RUTZ, F. Effects of different dietary levels of crude protein and digestible energy on Performance of pejerrey fingerlings. Revista Brasileira de Agrociência, v.10, n.1, p. 97-101, 2004.

REIS, E.S.et al. Suplementação de vitamina C na dieta para larvas de jundiá Rhamdia voulezi. Ciência Animal Brasileira, v. 12, n. 1, p. 83-89, 2011.

ROTTA, M.A. Utilização do ácido ascórbico (vitamina C) pelos peixes. Corumbá: Embrapa Pantanal, 2003.54 pp.

SHIAU, S.Y. and HSU, T.S. Quantification of vitamin C requirement for juvenile hybrid tilapia, Oreochromis niloticus x Oreochromis aureus, with L-ascorbyl-2 monophosphate- na and Lascorbyl- 2-monophosphate-Mg. Aquaculture, v.175, p.317-326, 1999.

SOLIMAN, A.K.; JAUNCEY, K.; ROBERTS, R.J. The effect of dietary ascorbic acid supplementation on hatchability, survival rate and fry performance in Oreochromis mossambicus (Peters). Aquaculture, Amsterdam, v.59, n.3-4, p.197-208, 1986.

ZHOU, Q.et al. Effect of dietary vitamin C on the growth performance and innate immunity of juvenile cobia (Rachycentron canadum). Fish \& Shellfish Immunology, v.32, p.969-975, 2012. 